\title{
The Ambivalent Role of Glial Cells in Neuroinflammation and Neuropathic Pain
}

\author{
Bilal F Shanti ${ }^{*}$, Ihsan F Shanti ${ }^{2}$, Khalil Al-Abbadi ${ }^{3}$, Ali \\ Al Rjoub ${ }^{4}$ and Ali Otom ${ }^{5}$ \\ ${ }^{1}$ Omnia Pain Consultants, Pain Medicine, Phoenix, Arizona, USA \\ ${ }^{2}$ The American Clinic for Interventional Pain and Spine, Amman, Jordan \\ ${ }^{3}$ Senior Consultant, Physical Medicine and Neurorehabilitation, Amman, \\ Jordan \\ ${ }^{4}$ Department of Physical Medicine and Rehabilitation, Al Bashir Hospital, \\ Amman, Jordan \\ ${ }^{5}$ Royal Specialty Center for Spine and Musculoskeletal disorders, Amman, Jordan \\ *Corresponding Author: Bilal F Shanti, Omnia Pain Consultants, Pain \\ Medicine, Phoenix, Arizona, USA.
}

\author{
Received: May 24, 2020 \\ Published: May 29, 2020 \\ (C) All rights are reserved by Bilal F Shanti., \\ et al.
}

\begin{abstract}
The current clinical management of neuropathic pain remains a real and big challenge to clinicians.

The last 3 decades have witnessed an explosion of research in the molecular neurobiology of pain. The traditional belief that pain modulation is solely based on peripheral and central pain pathways or based on peripheral pain modulators such as bradykinin, substance $\mathrm{P}$ and prostaglandin, is becoming secondary and redefined, albeit still valid. The novel look emphasizes on the role of glial cells (Mainly microglia and astrocytes) in the dorsal horn of the spinal cord, and their intricate communication with neurons. Similarly, the presumed passive physiologic role of glial cells being simply related to housekeeping like supporting, maintaining, repairing, nurturing, and protecting the neurons is becoming a mediocre definition. Glial cells, especially microglia, once activated, are found to orchestrate the initiation of pain processes, whereas astrocytes are responsible for pain maintenance. Once triggered, glial activation causes proliferation, morphological changes, and expression of receptors and markers (e.g. glial fibrillary acidic protein (GFAP)) that lead to the production inflammatory mediators (e.g. tumor necrosis factor- $\alpha$, interleukin-1 $\beta$, interleukin-6 (IL-6), nerve growth factor (NGF)), growth factors (brain-derived growth factor (BDNF) and basic fibroblast growth factor (bFGF)), cytokines and chemokines (e.g. MCP-1, CX3CL1, CCL2, CXCL13, CXCR5). This activation can have severe and deleterious effects on pain symptoms and trigger and maintain a serious pain syndrome. This ambivalent function of glial cell activation is the focus of our review manuscript. We feel it is important to understand these mechanisms as these may shed light on potential novel treatment options of neuroinflammation and neuropathic pain. It is important to understand that we have based our review on research on animals and the same may or may not apply for humans, and we have focused mainly on microglia and astrocytes.
\end{abstract}

Keywords: Glia; Microglia; Astrocytes; Neuropathic Pain; Pain; Neuroinflammation; Chemokines; Cytokines; Glial Activation

\section{Introduction}

International Association for the Study of Pain defines neuropathic pain simply as pain "caused by a lesion or disease of the somatosensory nervous system” [1].
Neuropathic pain is highly debilitating, disabling, and chronic. It poses a huge economic burden and affects millions of people worldwide. It is associated with serious psychosocial ramifications and affects the quality of life and productivity. The current treatment options for neuropathic pain are limited in quality and side effects. This poses a serious challenge on the treating clinician. 
From our basic physiology knowledge, we know there are way more glial cells in the brain than there are neurons. Actually, glial cells are the most abundant cells in the nervous system. Yet our traditional belief that neurons played all the higher functions remained valid for decades. In 1961, Galambos challenged that theory. We now know that glial cells work in harmony with neurons as one unit structured as 'glial cells-neurons' [2] and constant communication in a neuronal-glial signaling.

Glial cells are divided into 2 types, microglial cells (up to $10 \%$ of glial cells) and macroglia cells that include astrocytes and oligodendrocytes [3]. Unlike neurons, glial cells do not convey nerve impulses. When non-activated, their function is more of auxiliary maintenance, policing, and regulation of the neuronal microenvironment. They help in the scavenging of dead neurons, regulation of the brain vasculature and blood-brain barrier. Once activated, glial cells do become "short-tempered", causing detrimental pain problems, and this is the main focus of this manuscript.

The authors feel that practicing clinicians should be aware of the basic molecular neurobiology of neuropathic pain and its cascade. By assimilating this, they will realize the reasons it is so a challenge to treat this entity.

\section{A quick review of physiologic and pathologic pain signaling}

Like glial cells, pain itself is an ambivalent symptom. It is beneficial because it lets us know that there is a problem or injury to a particular tissue or nerve. If it becomes chronic and severe, it transforms into a debilitating disease. Our basic universal knowledge of pain signaling dictates that most tissues in the human body have nociceptors and these are receptors for pain stimuli. A $\alpha$ and $A \beta$ myelinated fibers respond to touch, light and vibration. The pain receptors are either lightly myelinated and called $A \delta$, or not myelinated and are called C fibers. Many neuromodulators and neurotransmitters are synthesized and released by the nociceptive sensory neurons from their terminals within the dorsal horn. These substances include brain-derived neurotrophic factor (BDNF), glutamate, galanin, calcitonin gene related peptide (CGRP), substance P (SP), adenosine triphosphate (ATP), vasopressin, somatostatin and vasoactive intestinal peptide (VIP). Once in the dorsal horn, nociceptive signals cross-over to the other side of the cord to the thalamic nuclei. From there, signals will travel to the brain stem and midbrain, including areas such as locus coeruleus, the periaqueductal gray (PAG) and the rostral ventromedial medulla (RVM).
From the thalamus, tertiary neurons convey the nociceptive signal to specialized areas in the cerebral cortex where the pain sensation is formed.

If there is peripheral tissue damage, inflammatory cascades fire up and 'peripheral sensitization' occurs. This process involves tumor necrosis factor (TNF), IL-1 $\beta$, prostaglandins (PG), bradykinin, nitric oxide (NO), ATP, and neurotrophins (BDNF, NT-3, NT-4, NGF). There is concurrent release of these mediators in the spinal dorsal horn thus amplifying nociception at the brain [4]. This 'spinal sensitization' is mediated by the release of NO, SP, PG, glutamate and other substances. This phenomenon is explained by many mechanisms that include, long term potentiation (LTP) in dorsal horn neurons [5], increase in peptide content, alterations in the expression of peptide receptors and sodium channels in sensory neurons [6], altered AMPA receptor expression in dorsal horn, increased facilitatory tone in descending pain pathways [7] and disinhibition in descending pathways or intrinsic neurons at segmental levels [8].

In dorsal horn, spinal sensitization involves activation of glial cells and BDNF mainly is implicated in increasing pain perception [8]. Similarly, astrocytes activation is implicated as a cause in persistence of pain [9].

\section{Neuroinflammation}

Neuroinflammation is a normal and expected reactive process. When controlled, as in the 'restive' acute phase after an injury, it is self-limited and under check. Once it enters, the chronic phase or in case of autoimmune diseases, it can manifest itself as reactive, severe, debilitating, hard to treat entity. Its hallmark is chronic inflammation. Its persistence leads to release of chemokines, cytokines, neuroimmune activation, and glial cells activation. If not controlled, this activation can lead to the infiltration of immune cells into the site of injury in response to a peripheral or central insult [10].

Once glial activation occurs, substances released from the injured site to the blood affect the blood-brain barrier and cause an increased permeability of the tight junctions of the capillary endothelial cells. This allows passage of blood cells into the CNS [11]. Because of their strategic positioning between the vessels and synapses, astrocytes control and monitor the neuronal activity and transmitter release. Increased release of glutamate and ATP leads to disturbances in $\mathrm{Ca}^{2+}$ signaling, increased production of cytokines 
and free radicals, attenuation of the astrocyte glutamate transport capacity, and changes in the astrocytic morphology, the actin filaments, which can cause the genesis and formation of new synapses [11]. These new neuronal contacts are created for maintaining and spreading pain sensation with the astrocytic networks as bridges. Therefore, glial cells can cause persistence of pain sensation even after the original injury has disappeared and turn this pain into chronic persistent type by altering the neuronal excitability [11].

We must remember that neuroinflammation is not a simple process. It is a complex integration of all cells of the CNS, including microglia, astrocytes, and the infiltrating leukocytes. This complex neuroinflammatory unit is determined by many factors such as the initial insult, genetics and environmental factors, past experiences, age, and many more factors that we don't know at this stage [12].

\section{What defines glial cells}

As aforementioned, glial cells represent the most abundant cells in the nervous system. Broadly speaking, there are two types of glial cells, microglia and macroglia. Microglia constitute 5 - 10\% of the entire glial cells whereas macroglia (including astrocytes and oligodendrocytes) represent the rest [13]. Depending on their locations, they are also classified as occurring either in the peripheral nervous system (satellite glia, perineural glia, Schwann cells) or in the central nervous system (microglia, astrocytes, oligodendrocytes, perivascular glia) [14].

For decades, glial cells role was under-estimated and was only thought to be limited to supporting neurons in their development, function, repair, scavenging, and maintenance. In the last two decades, a surge in interest has shed light on their role in initiating (microglia) and maintaining pain (astrocytes) [15]. Their ambivalent role permits microglia to either protect or harm neurons depending on the location and mechanism of their activation [15].

\section{Glial cells activation}

Chronic pain persistence and neuropathic pain chronicity account, in part, for the glial activation and the neuro-glial interactions. Glial cell activation in this manuscript refers mainly to the activation of microglia and astrocytes. The role of the other glial cells (Schwann cells and satellite cells) will also be mentioned below, but not in a detailed fashion, as it is not the focus of this paper.

\section{States of glial cell activation}

There are 4 identified types of pathway states of glial activation: (1) glial reaction with concurrent upregulation of glial markers such glial fibrillary acidic protein (GFAP) and IBA1 and hypertrophic proliferation and modification of glial networks, (2) phosphorylation of mitogen-activated protein kinase (MAPK) signal pathways, (3) upregulation of adenosine triphosphate (ATP) and chemokine receptors and downregulation of glutamate transporters, and (4) synthesis and release of glial mediators such as cytokines, chemokines, neurotrophins and other growth factors, and proteases to the extracellular space [16].

\section{Glial cell activation markers}

Activation of glial cells can be monitored by their expression of specific markers and kinases, changes in their morphology, and the release of specific immune substances [17]. This is important as it will inform us when the glial activation takes place and clinically potentially as a marker that pain is becoming chronic and debilitating. Once activated, microglia and astrocytes hypertrophy and increase the production of cellular proteins and cell surface markers [18]. Activated microglia induce markers as CD11b (cluster differentiation $11 \mathrm{~b}$, integrin $\alpha \mathrm{M}$, Mac- $1 \alpha$ ) and marker Iba1. Microglial markers also include MAPK [19], extracellular signal-regulated kinases (ERK) [20], glial glutamate transporters [21] and spinal gap junction proteins [22], toll-like receptors [23] and the complement component [24]. On the other hand, astrocyte activation induces and increase in GFAP and S100 $\beta$ [25].

\section{Glial cell activation}

Glial cells compromise 5 - $10 \%$ of the cells in the CNS. They stem from bone marrow-derived monocytes migrating during perinatal time [26]. In resting state, few glial cells divide or display hypertrophy. Their proliferation only starts after an insult such as a nerve injury [27]. Spinal cord glial activation is a common denominator for many pain syndromes including peripheral nerve damage, spinal cord inflammation, diabetic neuropathies, chemotherapy-induced neuropathies [28].

Further details on the cascade of glial activation and the release of proinflammatory cytokines was described above under abnormal pathologic pain signaling.

\section{Astrocytes activation}

Astrocyte role in chronic pain is paramount. As previously mentioned, unlike microglia that initiate pain, astrocytes sustain pain 
and make it persist. Also, once activated, astrocytes exhibit multiple morphological and functional changes after noxious insult and injuries. These transformations include (1) morphological changes as in hypertrophy, (2) proliferation, (3) gene expression changes, (4) significant molecular changes and (5) functional changes [29].

We now know there are at least 2 types of activate astrocytes: type A1 and type A2 [30]. A1 astrocytes, are triggered by neuroinflammation, produce neurotoxins that cause rapid neurons death and oligodendrocytes. On the other hand, A2 astrocytes are triggered by ischemia and promote neuronal survival and tissue repair [30]. We also mentioned that once under stress of an injury, astrocyte activation induces and increase in GFAP and S100 $\beta$ [25]. Both of these are markers reflect their surge and activation. In addition, two types of reactive astrocytes can be identified according to their individual genetic expressions. Complement 3 (C3), CFB and MX1S are the characteristic and solely upregulated genes in A1 astrocytes and are not expressed in A2 astrocytes [30]. On the other hand, the S100 protein family member S100A10 has been identified as specific markers of A2 astrocytes [31].

What prompts the resting astrocyte to convert from restive to reactive/activated phase is determined by multiple signaling molecules that can be released by many cell types, including neurons and glial cells, oligodendrocytes, astrocytes, and inflammatory cells [32]. The released signaling molecules include proinflammatory cytokines (IL-1 $\beta$, TNF- $\alpha$, and IL-6), oligodendrocyte transcription factor 2 (OLIG2), extracellular signal-regulated kinase 1/2 (ERK1/2), Sma- and Mad-related protein (SMAD) and G1 to $\mathrm{S}$ phase transition 1 (GSPT1), gene transcription factors (signal transducer and activator of transcription 3 (STAT3), and proteins (GFAP, connexins, and aquaporin 4 (AQP4)) [33]. Regardless whether their role is mediated by activated astrocytes or not, these signaling molecules are also involved in the development of chronic pain [29]. Whether they bypass or depend on activated astrocytes remains unknown.

\section{Activation of p38 MAPK and neuropathic pain}

As mentioned earlier, there are 4 types of states of glial activation. One of them, the phosphorylation of mitogen-activated protein kinase (MAPK) signal pathway, seems to be directly connected to regulating neural plasticity and inflammatory response, and hence pain. The MAPK family itself consists of three main types: extracellular signal-regulated kinases (ERK), p38 and C-Jun
$\mathrm{N}$-terminal kinase (JNK) [33]. ERK activation in the dorsal horn of the spinal cord and amygdala plays an essential role in central sensitization by controlling glutamate receptors and $\mathrm{K}$ channels and inducing gene transcription [33]. Once glial cells are activated secondary to a nerve injury, ERK, p38 and JNK get differentially activated as well and lead to the synthesis of pronociceptive and proinflammatory mediators (such as IL-1 $\beta$, IL-6, TNF $\alpha, \mathrm{PGE}_{2}, \mathrm{NO}$, NGF, etc.) and the result is pain enhancement and persistence [33].

The kinase p38 MAPK plays a critical role in inflammatory responses. The p38 inhibitor was shown to effectively alleviate rheumatoid arthritis and inflammatory pain $[34,35]$. In addition to neuropathic pain, microglial activation of p38 was also linked other pain conditions [36].

\section{Neuropathic pain link to neuroinflammation}

Presently, we know that microglial activation initiates early neuropathic pain and astrocytes make it persist [37]. At the spinal cord level, glial activation is sufficient for persistence of pain states associated with diabetic neuropathy, chemotherapy-induced neuropathy, peripheral nerve inflammation and trauma and spinal cord inflammation [38].

We now know that glial cells activation triggers the differentiation and activation of ERK, p38 and JNK and this leads to the synthesis of pronociceptive and proinflammatory mediators (such as IL-1 $\beta$, IL-6, TNF $\alpha, \mathrm{PGE}_{2}, \mathrm{NO}, \mathrm{NGF}$, etc.) and the result is pain enhancement and persistence [33].

\section{Cytokines and chemokines}

White., et al. suggested that chemokines play important roles in microglia activation and pain sensitization [39]. As an example, CXCL1 is produced in dorsal root ganglion (DRG) neurons and is able to cause pain hypersensitivity [40]. Another chemokine, CX3CR1, is only expressed in spinal microglia and upregulated after nerve injury [40]. When a neutralizing antibody to CX3CR1 was injected intrathecally, it was able to inhibit neuropathic pain $[40,41]$ and inflammatory pain [42].

Recent research by Zhang., et al. done in 2017, has shown that in case of chronic painful conditions, there is expression, distribution and function of chemokines in the spinal cord [43]. This monumental research demonstrated the upregulation of many chemokines after the peripheral nerve injury and with different levels of 
neuron-glia interaction in the spinal cord [43]. For example, the first finding as a proof of neuron-to microglial signaling, CX3CL1 expressed while using receptor CX3CR1 in primary afferent and spinal neurons and caused microglial activation. Second, as astrocyte-to-neuron signaling, CCL2 and CXCL1 expression on spinal astrocytes, using spinal neurons receptors CCR2 and CXCR2, increased the excitatory synaptic transmission. Thirdly, demonstrating neuron-to-astrocytes signaling, CXCL13 highly upregulated in spinal neurons and induced spinal astrocytes activation via receptor CXCR5 [43]. This cascade highlights a potential treatment targeting chemokine mediated neuron-glial activation.

\section{The other glial cells: Schwann cells and satellite cells}

The principal glial cells of the peripheral nervous system are the Schwann cells. In myelinated neurons, Schwann cells form the myelin sheath. Non-myelinating Schwann cells are involved in maintenance of axons and are crucial for neuronal survival. Schwann cells forming myelin sheath around A-fibers and non-myelinating Remak bundles around nociceptive C fibers do react to peripheral insults.

Schwann cell activation results in modification of myelin properties altering the conduction properties of nociception [44]. In addition, Schwann cells release proteases that may cause interference with blood-nerve-barrier thus allowing the circulating immune cells to enter the nerve and cause nociception-facilitation actions [45].

Satellite glial cells (SGC) are glial cells that cover the neuron cell bodies in ganglia of the peripheral nervous system. SGC are well located to envelope sensory neurons within the spinal and trigeminal ganglia. In a sense, they act like astrocytes by keeping a check on neuronal activity and exert regulatory functions [46].

Pertin., et al. found that following a nerve injury, SGC upregulate the synthesis of neurotrophins acting both as promoters of sympathetic sprouting within the ganglion [47] and as direct sensitizers of nociceptive neurons [48].

In a study done by Liu., et al. in 2012, using GFAP as a marker for SGC activation (as in astrocyte activation) the team demonstrated that SGCs in DRG were activated after L5 spinal nerve ligation. When they applied fluorocitrate, a glial metabolism inhibitor, into L5 DRG it significantly decreased the mechanical allodynia. SGC ac- tivation contributed to the early maintenance of allodynia after the spinal nerve lesion [49]. This points to a role of SGC activation in early neuropathic pain. However, not much is known on how SGC sensitize sensory neurons.

\section{Glial cell activation in humans}

In general, experimental research on glial cell, neuroinflammation and neuropathic pain, has been solely limited to the animal model. Whether these findings apply to humans or not is a different story. Typically, the animal model molecular neurobiology is similar but not identical to the humans. However, research is still lacking in this field and there is no confirmed correlation that biomarkers increase is associated with chronic pain.

Del Valle., et al. has studied spinal cord histopathological changes in humans afflicted with complex regional pain syndrome (CRPS) [50]. Using immunohistochemistry on spinal cord of autopsy patients, the team studied the impact of glial cell activation and the neuronal loss in the cervical, thoracic, and lumbar spinal cord of patients with advanced CRPS. They compared these to the spinal cord of 4 control patients. They found evidence of posterior horn cell loss and activation of both astrocytes and microglia, concentrated mainly at the site of injury, although spread throughout the whole spinal cord [50]. This duplicated finding, from animals to humans, may demonstrate that the findings in the animal model may apply to humans as well, but this remains to be clarified.

Similarly, Brisby., et al. studied the cerebrospinal fluid of patients with lumbar disk herniation and sciatica. They were looking for markers for glial cells. They found that these patients had increased concentrations of neurofilament protein and S-100 in the cerebrospinal fluid, which indicates damage of axons and Schwann cells in the affected nerve root [51].

Yet in another study conducted on children with chronic migraine headaches, wanted to see if the biomarkers increase with this chronic pain. The findings from blood samples suggested a direct relation between childhood migraine attacks and increased production of glial S100beta protein [52].

These findings tell us that the experiments conducted on rodents were somewhat duplicated in humans.

\section{Possible future novel treatments}

The last 2 - 3 decades were happy years for neuroscience and molecular neurobiology as related to pain. Major discoveries were 
made in form of markers, pathways, receptors, neurotransmitters, peripheral/central sensitization and glial activation. Unfortunately, whether this will cause an immediate improvement of treatment of neuropathic pain or not is doubtful. Presently, the medications that are available to treat neuroinflammatory pain and neuropathic pain still carry high toxicity, are short-lived, have poor coverage, and are not target-specific.

In the future, we predict the development of specific glial inhibitors that will be able to prevent and maybe even reverse glial phenotypes and expression, and even agents that prevent hypertrophy of astrocytes and their expression.

New anti-inflammatory agents will be hopefully discovered, as many are in the pipeline. These will likely tackle the issue of neuroinflammation and its ramifications. We are hoping that specific inhibitors to neurotransmitters and markers, and even antidotes to certain immune cells may be discovered soon.

Similarly, agents that target and reverse the glial intracellular mediators such as MAPKs, p38, JNK will decrease the prevalence of neuropathic pain and central sensitization. Additionally, agents that will target chemokines and cytokines and prevent chemokinemediated neuron-glial interactions may lead to novel treatments of neuropathic pain. Possible discovery of selective inhibitors of proinflammatory and pronociceptive substances, such as IL-1 $\beta$, BDNF, growth factors, and TNF will be useful in the treatment of pain.

\section{Conclusion}

Neuroinflammation and neuropathic pain are still major clinical burden to patients, their families, clinicians, to the economy, and the society as a unit. The last 3 decades have witnessed amazing new discoveries that champion the field of pain medicine and pain neurobiology. Nevertheless, a lot of research still needs to be done.

We now know that the traditional belief of pain modulation that is solely based on peripheral and central pain pathways, or based on peripheral pain modulators such as bradykinin, substance $\mathrm{P}$ and prostaglandin, is becoming outdated. Similarly, glial cells being simply related to housekeeping like supporting, maintaining, repairing, nurturing and protecting the neurons is becoming a history of the past. Research trends are now focused on glial activation. This activation causes proliferation, morphological changes, and expression of receptors and markers that leads to chronic pain.
Potential discovery to target each of these will be extremely useful.

The present experimental research negates the role of the onceassumed dormant role of glial cells. Presently, glial cell activation, astrocytes activation and expression, the role of cytokines and chemokines, neurotrophins, proinflammatory and pronociceptive mediators, activation biomarkers, the prospective role of the other glial cells such as Schwann cells and satellite cells, receptors, pathways, all are potential target for novel therapies.

Reliable human data is paramount in this field. This is necessary to make sure the animal model findings are no different in humans.

We owe it to our patients to uphold the pledge of "decreasing pain and suffering".

\section{Conflict of Interest Disclosure}

All the authors disclose they have no conflict of interest.

\section{Author's Contribution}

All authors contributed equally to this work.

\section{Bibliography}

1. International Association for the Study of Pain. IASP Taxonomy. Pain terms. Neuropathic pain (2017).

2. Galambos R. "A glia-neural theory of brain function". Proceedings of the National Academy of Sciences of the United States of America 47.1 (1961): 129-136.

3. Moalem G and Tracey DJ. "Immune and inflammatory mechanisms in neuropathic pain". Brain Research Reviews 51 (2006): 240-264.

4. Latremoliere A and Woolf CJ. "Central Sensitization: A Generator of Pain Hypersensitivity by Central Neural Plasticity". The Journal of Pain 10.9 (2009): 895-926.

5. Ikeda H., et al. "Synaptic Plasticity in the Spinal Dorsal Horn". Journal of Neuroscience Research 64.2 (2009): 133-136.

6. Yaksh TL. "Central pharmacology of nociceptive transmission". Elsevier, Churchill Livingtone (2006).

7. Ren K and Dubner R. "Pain Facilitation and Activity-Dependent Plasticity in Pain Modulatory Circuitry: Role of BDNF-TrkB Signaling and NMDA Receptors". Molecular Neurobiology 35 (2007): 224-235 (2007). 
8. Coull, J., et al. "BDNF from microglia causes the shift in neuronal anion gradient underlying neuropathic pain". Nature 438 (2005): 1017-1021.

9. Garrison CJ., et al. "Staining of glial fibrillary acidic protein (GFAP) in lumbar spinal cord increases following a sciatic nerve constriction injury". Brain Research 565.1 (1991): 1-7.

10. Baptista MJ., et al. "Parkin and alpha-synuclein: opponent actions in the pathogenesis of Parkinson's disease". Neuroscientist 10.1 (2004): 63-72.

11. Hansson E. "Long-term pain, neuroinflammation and glial activation”. Scandinavian Journal of Pain1.2 (2010).

12. Carson MJ., et al. "The cellular response in neuroinflammation: The role of leukocytes, microglia and astrocytes in neuronal death and survival". Clinical Neuroscience Research 6.5 (2006): 237-245.

13. Moalem G and Tracey DJ. "Immune and inflammatory mechanisms in neuropathic pain". Brain Research Reviews 51.2 (2006): 240-264.

14. Kriegstein A and Alvarez-Buylla A. "The glial nature of embryonic and adult neural stem cells". The Annual Review of Neuroscience 32 (2009): 149-184.

15. Jha MK., et al. "Glia as a Link between Neuroinflammation and Neuropathic Pain". Immune Network 12.2 (2012): 41-47.

16. Ji RR., et al. "Glia and pain; is chronic pain a gliopathy?" The Journal of Pain 154.1 (2013): S10-S28.

17. DeLeo JA., et al. "Immune and glial regulation of pain". Seattle: IASP Press (2007).

18. Ren K and Dubner R. "Neuron-glia crosstalk gets serious: Role in pain hypersensitivity". Current Opinion in Anesthesiology 21.5 (2008): 570-579.

19. Wen YR., et al. "Nerve conduction blockade in the sciatic nerve prevents but does not reverse the activation of p38 mitogenactivated protein kinase in spinal microglia in the rat spared nerve injury model”. Anesthesiology 107 (2007): 312-321.

20. Zhuang ZY., et al. "ERK is sequentially activated in neurons, microglia, and astrocytes by spinal nerve ligation and contributes to mechanical allodynia in this neuropathic pain model". Pain 114.1-2 (2005): 149-159.

21. Sung B., et al. "Altered expression and uptake activity of spinal glutamate transporters after nerve injury contribute to the pathogenesis of neuropathic pain in rats". The Journal of Neuroscience 23.7 (2003): 2899-2910.

22. Spataro LE., et al. "Spinal gap junctions: potential involvement in pain facilitation". The Journal of Pain 5.7 (2004): 392-405.

23. Tanga FY., et al. "The CNS role of Toll-like receptor 4 in innate neuroimmunity and painful neuropathy". Proceedings of the National Academy of Sciences of the United States of America 102.16 (2005): 5856-5861.

24. Griffin RS., et al. "Complement induction in spinal cord microglia results in anaphylatoxin C5a-mediated pain hypersensitivity". The Journal of Neuroscience 27 (2007): 8699-8708.

25. Tanga FY., et al. "Role of astrocytic S100beta in behavioral hypersensitivity in rodent models of neuropathic pain". Neuroscience 140.3 (2006): 1003-1010.

26. Nakajima K and Kohsaka S. "Microglia: activation and their significance in the central nervous system". Journal of Biochemistry 130.2 (2001): 169-175.

27. Graeber MB., et al. "Microglial cells but not astrocytes undergo mitosis following rat facial nerve axotomy". Neuroscience Letters 85.3 (1988): 317-321.

28. Watkins LR., et al. "Chapter 22 Contribution of glia to pain processing in health and disease". Handbook of Clinical Neurology 81 (2006): 309-323.

29. Li T., et al. "An update on reactive astrocyte in chronic pain". Journal of Neuroinflammation 16 (2019): 140.

30. Liddelow SA., et al. "Neurotoxic reactive astrocytes are induced by activated microglia". Nature 541.7638 (2017): 481-487.

31. Fujita A., et al. "Connexin 30 deficiency attenuates A2 astrocyte responses and induces severe neurodegeneration in a 1-methyl-4-phenyl-1,2,3,6-tetrahydropyridine hydrochloride Parkinson's disease animal model". Journal of Neuroinflammation 15.1 (2018): 227. 
32. Pekny M., et al. "Astrocytes: a central element in neurological diseases". Acta Neuropathologica 131.3 (2016): 323-345.

33. Ji RR., et al. "MAP kinase and pain". Brain Research Reviews 60.1 (2009): 135-148.

34. Kumar S., et al. "p38 MAP kinases: key signalling molecules as therapeutic targets for inflammatory diseases". Nature Reviews Drug Discovery 2 (2003): 717-726.

35. Ji RR., et al. "p38 MAPK activation by NGF in primary sensory neurons after inflammation increases TRPV1 levels and maintains heat hyperalgesia". Neuron 36 (2002): 57-68.

36. Cui Y., et al. "Activation of p38 mitogen-activated protein kinase in spinal microglia mediates morphine antinociceptive tolerance". Brain Research 1069 (2006): 235-243.

37. Raghavendra V., et al. "Complete Freunds adjuvant-induced peripheral inflammation evokes glial activation and proinflammatory cytokine expression in the CNS". European Journal of Neuroscience 20.2 (2004): 467-473.

38. DeLeo JA and Winkelstein BA. "Physiology of chronic spinal pain syndromes: from animal models to biomechanics". Spine 27.22 (2002): 2526-2537.

39. White FA., et al. "Chemokines and the pathophysiology of neuropathic pain". Proceedings of the National Academy of Sciences of the United States of America 104.51 (2007): 20151-20158.

40. Verge GM., et al. "Fractalkine (CX3CL1) and fractalkine receptor (CX3CR1) distribution in spinal cord and dorsal root ganglia under basal and neuropathic pain conditions". European Journal of Neuroscience 20.5 (2004): 1150-1160.

41. Zhuang ZY., et al. "Role of the CX3CR1/p38 MAPK pathway in spinal microglia for the development of neuropathic pain following nerve injury-induced cleavage of fractalkine”. Brain, Behavior, and Immunity 21.5 (2007): 642-651.

42. Sun S., et al. "New evidence for the involvement of spinal fractalkine receptor in pain facilitation and spinal glial activation in rat model of monoarthritis". Pain 129.1-2 (2007): 64-75.

43. Zhang ZJ., et al. "Chemokines in neuron-glial cell interaction and pathogenesis of neuropathic pain". Cellular and Molecular Life Sciences 74.18 (2017): 3275-3291.
44. Gosselin RD., et al. "Glial cells and chronic pain". Neuroscientist 16.5 (2010): 519-531.

45. Scholz J and Woolf CJ. "The neuropathic pain triad: neurons, immune cells and glia". Nature Neuroscience 10.11 (2007): 1361-1368.

46. Hanani M. "Satellite glial cells in sensory ganglia: from form to function". Brain Research Reviews 48.3 (2005): 457-476.

47. Pertin M., et al. "Delayed sympathetic dependence in the spared nerve injury (SNI) model of neuropathic pain". Molecular Pain 3 (2007): 21.

48. Zhou XF., et al. "Satellite-cell-derived nerve growth factor and neurotrophin-3 are involved in noradrenergic sprouting in the dorsal root ganglia following peripheral nerve injury in the rat". European Journal of Neuroscience 11.5 (1999): 1711-1722.

49. Liu FY., et al. "Activation of satellite glial cells in lumbar dorsal root ganglia contributes to neuropathic pain after spinal nerve ligation". Brain Research 1427 (2012): 65-77.

50. Del Valle L., et al. "Spinal cord histopathological alterations in a patient with longstanding complex regional pain syndrome". Brain, Behavior, and Immunity 23.1 (2009): 85-91.

51. Brisby H., et al. "Markers of nerve tissue injury in the cerebrospinal fluid in patients with lumbar disc herniation and sciatica". Spine 24.8 (1999): 742-746.

52. Papandreou 0., et al. "Serum S100beta protein in children with acute recurrent headache: a potentially useful marker for migraine". Headache 45.10 (2005): 1313-1316.

\section{Assets from publication with us}

- Prompt Acknowledgement after receiving the article

- Thorough Double blinded peer review

- Rapid Publication

- Issue of Publication Certificate

- High visibility of your Published work

Website: www.actascientific.com/

Submit Article: www.actascientific.com/submission.php

Email us: editor@actascientific.com

Contact us: +919182824667 